\title{
Experience of Monocular Vision in Australia
}

Nicholas Buys and Jorge Lopez

Abstract: In-depth interviews with 20 people with monocular vision identified a number of themes associated with the impairment, including psychological, psychosocial, physical, vocational, and daily living problems. They also revealed that in many cases, these problems had profound implications for people's adjustment to daily living. The implications of these findings for the delivery of rehabilitation services are discussed.

People with monocular vision have no vision in one eye and typical vision in the other eye, with the consequent loss of binocular depth perception and a reduced field of vision. Monocular vision has received little attention in the disability and rehabilitation literature, and rehabilitation professionals often discount its economic and psychosocial consequences, viewing it as an inconvenience, rather than as a disability (Schein, 1988). Therefore, people with monocular vision are unlikely to be referred for rehabilitation services and thus are poorly prepared for the difficulties they experience in everyday life. 
Literature reviews have confirmed that most authoritative rehabilitation textbooks do not discuss the rehabilitation of people with monocular vision (Linberg, Tillman, \& Allara, 1988; Schein, 1988). This situation has made it difficult for rehabilitation practitioners to effectively identify and understand the needs of people with this impairment, leaving them poorly prepared to implement relevant rehabilitation programs. It has also perpetuated the fallacy that monocular vision is not a major disability and that rehabilitation is unnecessary (Schiff, 1980).

In contrast, the limited research in this area has found that people with monocular vision experience a range of perceptual problems that create difficulties in managing daily activities in such areas as mobility, work, prospects for employment, and living skills (Brady, 1994; Cooper, 2000; Gilberg, 1998; McKnight, Shinar, \& Hilburn, 1991; O'Donnell \& Smith, 1994; Schein, 1988; Schiff, 1980; Servos, Goodale, \& Jakobson, 1992; Spring, 1992). People with this condition also experience high levels of fatigue and susceptibility to illness and stress, resulting from the continuous effort required to adjust to monocular vision (Schein, 1988). Knoth (1995) reported that students with monocular vision commonly experience difficulty reading and studying that affects both their educational performance and their physical and emotional well-being. 
One area that has received little attention is the psychological and psychosocial sequelae of monocular vision. Brady (1994) found that people with this impairment experience a range of emotional reactions to their condition, ranging from "What is the use of living?" to "I hardly noticed the difference" (p. 17). Guilt and depression are commonly associated with monocular vision when people believe that the disability is "divine punishment" for past sins. By overcompensating for the condition, people may place themselves under such a high level of prolonged stress that psychiatric help may be needed (Schein, 1988). Emotional responses are often exacerbated by feelings of the loss of normality because of changes in cosmetic appearance, the lack of understanding of the condition, and anxiety related to the fear of total blindness, and the subsequent overprotection of the good eye.

Posttraumatic personality changes resulting from the underlying stress associated with adjusting to monocular vision have also been reported (Schein, 1988). Family issues can compound these problems, particularly when friends and relatives do not fully understand the impact of monocular vision on the individual. Given that the lack of psychosocial adjustment is one of the most significant barriers to achieving positive rehabilitation outcomes (Putman \& Adams, 1992), this is an important area for service providers.

Monocular vision has received little attention in 
Australia. Statistics on its incidence are unavailable, few formal rehabilitation programs exist to address the specific needs of this group, and people with this condition are seldom referred for rehabilitation services. In addition, information on the functional implications of monocular vision is rarely made available in the immediate postoperative period by nursing or medical staff. When they are discharged from the hospital, people with monocular vision receive only limited support from community rehabilitation services, and rehabilitation practitioners often have only limited knowledge of the impact of this impairment.

In summary, monocular vision has received little attention in the rehabilitation literature or formal service delivery mechanisms despite its significant impact on peoples' lives. The study presented here attempted to redress the lack of knowledge in this area by addressing the following research question: What problems do people identify as having arisen from their experience of having monocular vision?

\section{Method}

\section{Participants}

The participants were 20 people with monocular vision, 10 men and 10 women (for the characteristics of the participants, see Table 1). Most participants (16) were recruited from the client database of Guide Dogs 
Queensland, a large provider of rehabilitation services for people with visual impairments. The remaining 4 participants were recruited through informal contacts. No predetermined number of participants was selected in advance for the study. Instead, new participants were recruited until no new data were forthcoming.

The participants ranged in age from 22 to 77, with a mean of 47 years. Age 18 was chosen as the minimum age for participation because one focus of the study was the impact of monocular vision on past, current, and future employment. The age of onset of monocular vision ranged from birth to 74 , with a mean of 36 years. The most common cause of monocular vision was eye trauma; only 1 participant had congenital monocular vision, which resulted from cataracts. Of the 20 participants, 16 had no vision in the affected eye, and 4 retained a minimal level of light perception in this eye. People with amblyopia were not included in the study because they have a sufficient level of vision in the affected eye to allow for depth perception.

\section{Procedures}

Data were collected using semistructured interviews. The interview questions were pilot tested with three people with monocular vision to check their clarity and understandability. Before the interviews were conducted, the participants were informed that their participation was voluntary and that they could withdraw from the study at any time. They were also 
advised that their real names would not be used when preparing transcripts and reporting results, and their permission to audiotape the interviews was obtained. At the start of each interview, the aim of the study was discussed, and the participant was asked to read and sign a consent form.

The interview questions were designed to obtain indepth information about problems arising from the experience of monocular vision (see Box 1 for the interview guide). All the participants were asked the same questions to ensure consistency in the information obtained and to allow for comparisons across participants (Patton, 1990). The duration of the interviews ranged from 1 to $1 \frac{1}{2}$ hours, during which time the participants were encouraged to express their experiences and feelings freely with regard to how monocular vision had affected their daily lives.

Data from the audiotapes were analyzed using a manual system of coding and thematic categorization (Neuman, 1997). This process consisted of a continuous rereading of transcripts to identify a large number of themes from the data (open coding), followed by the grouping of similar themes under related headings by identifying which tended to cluster and where linkages between themes occurred (axial coding). Major themes were then located in the data, as well as in particular cases that illustrated them, and provided comparisons and contrasts (selective coding). Following the data analysis, two focus groups of 
participants were held to provide "participant checks" of the identified themes. During these sessions, the results were reported, and the participants were asked to comment on the accuracy of the themes in representing their experiences.

\section{Results}

The purpose of the study was to identify issues that arise from the experience of monocular vision. Toward this end, a number of major themes were identified that were broadly classified as psychological, psychosocial, physical, vocational, and activities of daily living. The major themes and associated problems are summarized in Table 2.

To illustrate these themes, comments from the participants are included. The use of such comments helps to retain the integrity of the study by grounding the results in the language of the participants (Reinharz, 1979). To protect the participants' confidentiality, pseudonyms are used.

\section{Psychological problems}

Psychological issues focused on common emotional reactions that are associated with the loss of vision in one eye. These reactions included the initial shock and inability to accept the permanency of the loss, the fear of total blindness, the loss of self-esteem and personal worth related to an altered sense of normalcy, and 
feelings of inadequacy and perceived clumsiness in social situations.

The majority of the participants who experienced the loss of sight in one eye as adults expressed feelings of fear and anxiety about the future and an inability to relax and enjoy life. Greg, for example, stated: "I feel frustrated and unsure of the future. I feel angry and anxious about everything, and glare is a problem, of course. . . . I feel it has made life even more difficult for me." Common feelings included fear, insecurity, an inability to cope, and reduced independence associated with the potential for total blindness. Some participants wore protective eyeglasses to avoid damage to the "good" eye and avoided "hazardous activities," such as gardening and mowing. In some instances, these fears were pronounced, as is illustrated by Anne's comment: "I felt quite depressed and fearful of the future and in real need of psychiatric care. I think I even considered suicide a number of times when it just happened."

Fear extended to other areas. Some participants reported feeling scared when people touched them unexpectedly on the blind side in crowded areas, such as shopping centers, or at social events. Others were afraid of driving in busy or unknown environments and of walking in crowded or poorly lit areas.

The loss of personal worth and acceptance and their impact on relationships was a significant problem for some participants, particularly those who had 
experienced cosmetic changes as a result of losing the eyeball and the consequent use of a prosthetic eye. Sarah, who lost her eye as a child, stated:

I felt rejected by the other children and even by my teachers because of my prosthetic eye, which, I think, made me look different. I think that because of this rejection, I always felt that I was not good enough.

Pauline, who needed cosmetic surgery to restore her preaccident facial appearance, was afraid of being pitied by others:

I had to undergo a number of cosmetic interventions to restore my eye socket. This changed my appearance and created in me difficulties in establishing new relationships or even keeping old relationships because of the cosmetic changes that affected my self-esteem.

Many participants' feelings of no longer being "normal" resulted in low self-esteem and the fear that peers would not accept them, particularly during their teenage years. One stated: "I felt unworthy of any attention when I reached my teenage years. I used to say to myself, 'Who can love a person like me?' " Feelings of inadequacy persisted for the participants who experienced the onset of monocular vision as adults. These participants saw problems of psychosocial adjustment as playing a major role in their acceptance of monocular vision. Alice stated:

I lost my self-confidence. It affected my decision making, and I became insecure. It affected every area of my life. I think I became more insecure with age because of the high 
pressures of my management job. I felt depressed all the time after the loss of sight in my right eye.

Resentment of treating physicians who did not inform them of the symptoms associated with monocular vision, such as the loss of depth perception and a reduced field of vision, prior to surgery were common among the participants. Arith, who lost her eye as a result of complications from a cataract operation, stated: "I will never forgive my doctor! It was so cruel and irresponsible not to warn me about the complications of this surgery."

\section{Psychosocial problems}

The participants who experienced the impairment at birth or as children and those who acquired it as adults responded differently to monocular vision. Those who had monocular vision from birth or childhood were more likely to believe that functional problems associated with the impairment were part of their intrinsic makeup, rather than a result of the impairment per se. This belief was reinforced by negative experiences with peers in social situations, at school, and in sports activities. In contrast, the participants who acquired monocular vision as adults attributed their functional problems to the impairment, from which they expected to recover. When recovery did not occur, they inaccurately attributed their ongoing difficulties to other perceived medical or psychological problems because of the lack of appropriate 
information. For both children and adults, these reactions often had significant psychosocial consequences. Several participants reported decreased confidence and self-esteem in social situations and a reduced ability to perform a range of social skills, such as pouring drinks, shaking hands, maintaining eye contact, and walking safely in crowded areas. Those with cosmetic changes reported experiencing emotional difficulties and having reduced self-esteem in social situations to a greater extent than did those without cosmetic changes.

Several participants avoided social involvement, becoming withdrawn and socially isolated. For example, Frank moved from the city to live with his family on a farm in the country. He explained: "I felt so self-conscious. I avoided people and moved to live in the country to cope with life. I stayed away from people out of fear of them laughing at me because of my eye." Other participants reported a tendency to withdraw from social contacts because of their clumsiness in crowded environments. Because of the loss of depth perception and a reduced field of vision, they bumped into people, furniture, and other objects. Fatigue arising from attempts to compensate for their vision loss also reduced their motivation to participate in social events. As Bev noted: "I feel tired trying to concentrate all the time to make sense of thingsjudging distances and interpreting information."

The participants' ability to maintain social involvement 
was related, to some extent, to the level of support received from their family members and friends, the degree of change in their cosmetic appearance, their level of understanding of the problem, and their coping ability. For example, several participants coped better with social situations when they and their families were made aware that their problems were related to their loss of depth perception and reduced field of vision. They were then able to prepare themselves for social encounters through simple strategies, such as planning where they were going to sit at a table in a restaurant.

\section{Physical problems}

Physical problems centered on the mental and physical fatigue resulting from the greater effort needed to perform various tasks. Physical exhaustion was more prevalent among the participants who were actively involved in job-related and daily living activities, such as reading, shopping, driving, or visiting friends. Sustained concentration to judge distances and interpret environmental visual information with monocular vision was extremely tiring. As Bev put it:

I feel mentally fatigued, and my damaged eye wanders out of focus when I'm tired. Glare affects my eye greatly. I still have a little window in the affected eye with some light, which is badly affected by glare.

Similarly, Sarah stated: 
The problem is getting worse as I get older. I feel tired, dizzy, and unable to concentrate at work while working with computers. My neck continues to hurt because of the continuous turning of my head to help me with changing lanes when driving.

\section{Vocational problems}

Vocational problems included the impact of monocular vision on current employment and career goals, as well as reduced income resulting from the loss or change of employment. The loss of depth perception greatly affected the participants' ability to perform many jobrelated tasks, particularly in the trades area, such as using power tools and accurately measuring and cutting materials. It was also a safety issue in that the negotiation of crowded workshop environments was difficult and dangerous. Of the 20 participants, 9 reported changing their careers as a result of their monocular vision. As Frank said: "I was forced to stop working as a truck driver and had to take up farming to cope with life. I had difficulty driving a truck with one eye." Greg, who lost his sight in the left eye as a result of a flying piece of metal, was not able to return to his job as an automobile body repairer because of difficulties associated with performing certain tasks. He stated: "I can't return to work. I have problems with walking safely in the shop with my vision. . . I can't hit the chisel with the hammer to prepare metal parts for welding; it can be really unsafe."

The realization of "career dreams" was also affected. 
Carl, who lost his eye as a teenager, had his application to enter the Air Force rejected because of monocular vision. This rejection had apparently stopped him from following the family tradition:

Yes! [I was] definitely affected. ... [I] wanted to get into the Air Force, but after I lost my eye, I wasn't accepted. . . . That left me totally confused, not knowing what to do of myself in relation to employment. I lost direction! ... I was not interested in anything else.

Sarah, who lost her eye as a child, was rejected from a program in nursing studies because of her monocular vision. As she noted:

I wanted to be a nurse, and my mother talked to the nurse in charge, seeking information about what I had to do, and [the nurse] said that I wouldn't be allowed because I had vision in only one eye.

Many participants reported that their career difficulties did not seem to be understood by most vocational rehabilitation professionals. Karen stated:

It was interesting to me as an individual. ... On the one hand, my specialist [said that I'd] be fine, and on the other hand, my employer [made] a big deal about it. My therapist assisting my return to work [made] a big deal about it, and so across the board of professionals that I dealt with ... there was a broad range of modulated levels of importance placed on this disability. And for me, it was quite confusing. ... I didn't know where to place myself.

The financial impact of job loss was significant. Frank, who had to stop driving his truck, received 
unemployment benefits, which reduced his earnings by almost $60 \%$. Pauline lost her business as a result of the range of problems associated with monocular vision. Anne, a beautician, stated:

Yes, I have been affected financially because of monocular vision impairment! . . I I had to stop teaching because of problems with driving at night, and I had to employ new staff to assist me with fine detailed cosmetic work. ... I was also forced to charge less for jobs I could not complete.

Coping successfully with monocular vision at work was often related to the use of adaptations. For example, Robert was able to cope, despite problems with depth perception, by using adaptive equipment to assist him with his job as a cook.

\section{Activities of daily living}

Most of the participants described problems with a wide range of daily activities because of monocular vision, including driving, walking, personal grooming, and performing household chores, such as cutting, placing objects on benches, filling up containers, and pouring liquids. Problems with cooking, particularly when kitchen areas were poorly lit, were common.

Difficulty judging distances or not seeing objects on the affected side hampered activities like reaching, picking up objects, shaking hands, catching balls, and hanging out clothes. For example, Bev stated: 
It takes me two or three tries before I can grab a pen offered to me. I have developed some skills, but the problem is still there after all these years, particularly if I am tired or in a rush. I ask [people] to place things in my hand to solve the problem, but it is still happening after three years; it has not improved.

The participants also reported problems with personal grooming, particularly when applying makeup, inserting eyedrops, shaving, and putting toothpaste on a toothbrush. As Pauline put it:

My eye doesn't focus properly; the eyelid has dropped, and it is hard to apply mascara on the [eyelash of the] good eye when the other eye is damaged. I have difficulty applying eyedrops or putting eye shadow on the eyelid of my good eye because I can't see anything when I close the good eye.

All the participants reported learning skills to cope with these problems over time. For example, Jane learned to cope better with pouring: "I learned to touch the glass with the jug spout, and after that, pouring was easy. I don't know how I do it, but it's not a problem; I have never overfilled a glass with water or juice."

However, the participants also acknowledged that new environments, fatigue, or time pressures compounded their difficulties and that they constantly had to think before doing anything. Bev stated:

If I'm tired, I feel as if my affected side was death and that everything is harder to handle. ... If I don't sleep, things get worse; the lack of sleep affects my performance.

Problems never change. I have learned to live with them, but they always come back if I am in a new environment 
or if I am tired.

Driving was a major problem for most participants. Difficult activities included accurately judging distances between a car and other objects; backing up into a parking space; overtaking other cars; managing oncoming traffic; negotiating narrow roads; turning corners; coping with glare; and driving in the rain, at night, or at dawn. Loss of confidence with driving was common, particularly when the participants were in unfamiliar environments or driving unfamiliar cars. As Pauline noted:

I have problems driving into indoor parking lots because of the reduced level of light and the narrow spaces for driving and parking. My confidence with driving is reduced, and yes, it has caused me to experience a change of roles at home from being the main driver to being driven everywhere by other members of my family.

These difficulties led to driving restrictions or problems learning to drive. For example, Janice was not able to obtain a driving license: "I found a total lack of interest by all training schools that I approached to assist me with driving lessons after I made them aware of my monocular vision impairment."

Traveling as passengers caused some participants high levels of stress when the cars they were in were in close proximity to other cars during such maneuvers as turning, overtaking other cars, and parking. Karen described how she continuously asked a driver to stop, slow down, or keep a greater distance from the car in 
front because it was difficult for her to know if the driver was driving safely.

I frustrated drivers by shouting at them to stop [and] watch out. I arrived [at a destination] a nervous wreck. I thought we were going to smash against a car all the time. It was frightening to be a passenger [and] even worse for the driver. I finally had to look down for the rest of the trip to stop this problem.

The participants described a range of strategies to cope with driving. These strategies included slowing down; doubling checking distances; adding mirrors to their cars; and planning the time of day, the direction in which to drive, and the place to park (preferably in open spaces) prior to driving anywhere. The participants also asked their passengers to check the traffic. Driving lessons assisted some, particularly in identifying the extra blind spots caused by monocular vision. Pauline stated:

Training and information on driving skills are important, particularly if the eye was lost in a car accident, plus monocular vision problems. [There are] more blind spots with monocular vision when driving a car. Cars and pedestrians are difficult to see on the blind side.

All the participants reported mobility problems, including bumping into people, furniture, door frames, and other objects, particularly in unknown environments, poorly lit areas, areas with low contrast, or crowded situations. Other problems included managing stairs, especially when descending; walking in shaded areas; managing changes in gradients and 
ground surfaces; judging curb heights, and negotiating broken footpaths. Arith stated:

I am terrified on uneven ground or steps. I have to use flat shoes for security. I feel nervous outside the house, judging curb heights, and so forth. I have to be careful; it gives me the shakes to go out. I fear my mobility is very restricted. I fear that I may fall and hurt myself all the time.

Coping with crowds was another problem. To help manage their reduced field of vision, particularly in crowded environments like shopping centers and busy city streets, the participants reported walking with their spouses or friends to boost their confidence. As Robert said:

I keep my wife by my affected side in crowded areas, and I keep my child on the good side to avoid leading him into obstacles without knowing [I'm doing so]. It could be dangerous, but if I am walking with adults, I prefer them on the affected side.

Some participants preferred that people walk on their affected side to force themselves to scan toward this side while talking. However, others preferred having people walk on their "good" side because of the need to keep in continuous contact with them. The failure to do so often resulted in their walking alone and talking to themselves.

The maintenance of hobbies was sometimes affected by monocular vision because of the loss of depth perception and the reduced field of vision. Reading and 
gardening were some of the activities that were affected. As Pauline noted:

My flower arrangements are flat when looked at from the side or when seen at a distance; they have no artistic balance. I also have great difficulty with videotaping, since I find it difficult to walk around while having my good eye fixed in the viewer of the video camera.

Sports, such as tennis, cricket, baseball, and PingPong, that involve hand-eye coordination, speed, and small balls were extremely difficult to play because of the difficulty of catching and hitting. Several participants developed a range of skills to enable them to continue their hobbies. Carl switched to pool and darts, sports in which targets are fixed. Robert changed to golf and swimming, in which no hitting or catching fast-moving balls is required. John maintained his interest in woodworking because of the psychological benefits, although it took longer and it was harder for him to do things after he developed monocular vision. As he reported:

Activities helped me to overcome the emotional problems. Depth perception affects the woodwork; finding tools is more difficult, and when I try to pick them up from the workbench, I miss them all the time. Judging distances is a problem, but I have had to accept it; it has become a part of life. I have learned to live with it.

\section{Discussion}

People with monocular vision face significant difficulties because of their visual impairments. The 
findings of this study revealed a range of psychological, psychosocial, physical, vocational, and daily living problems that affect many areas of life and have profound consequences for people's psychological and physical well-being. Although some people with monocular vision are able to lead relatively typical lives, for many, the effects of the impairment can be pervasive. Fear, poor self-esteem, and feelings of inadequacy often contribute to depression and social isolation, which are compounded by the physical and mental fatigue caused by the exertion required to manage the demands of life with this condition. For some, the impact of monocular vision on work is devastating, leading to the loss of jobs and businesses, the inability to pursue career dreams, and reduced incomes. The performance of daily activities, such as driving, mobility, grooming, household chores, and hobbies and sports is also often adversely affected.

Although the findings of this study are similar to those reported in other countries, such as the United States (see, for example, Brady, 1994; Schein, 1988), two additional difficulties were identified. First, the participants who acquired monocular vision at birth or as children often attributed associated functional problems to an inherent deficiency in themselves, rather than to the impairment. This attribution had a negative psychosocial impact on their relationships with peers and self-confidence at school, in sports activities, and in social situations. In contrast, those 
who acquired monocular vision as adults were more likely to attribute functional problems to the impairment. However, if they had expectations of recovery from the condition and they did not recover, they then tended to attribute problems to another cause, such as a perceived medical or psychological condition. These perceptions had an adverse impact on their self-esteem and confidence, resulting in social withdrawal and isolation. Attributional differences between children and adults regarding the cause of functional problems and their psychosocial impact require further investigation.

The second additional problem that was identified in this study is the experience of people with monocular vision as passengers in, not just drivers of, cars. Difficulty judging distances between vehicles in which they are passengers and other vehicles or environmental features can lead to heightened anxiety among people with monocular vision. Given Australia's great reliance on automobiles as a major form of transportation, this anxiety can be sufficient to avoid travel, thereby contributing to further isolation.

The participants in this study differed in their adjustment to monocular vision, and although it is beyond the confines of this article to explore this issue in any depth, there appear to be some key factors that assist with adjustment. The first key factor is the support of family members and friends. The participants' ability to remain socially involved was 
enhanced when family members and friends understood what the participants were going through and encouraged them to remain connected with others. Furthermore, managing social interactions became easier when the participants were aware of the problems associated with monocular vision. The ability to explain to others the reason for their clumsiness helped them cope with social interactions.

The second key factor was the provision of accurate information by physicians about the functional implications of monocular vision. The lack of appropriate information at the time of diagnosis or discharge from the hospital caused the participants to become resentful and confused because they felt they were inadequately prepared to face the difficulties that would arise from the impairment and were uncertain that what they were going through was typical. This confusion appeared to extend to family members as well.

The third key factor is the inadequacy of communitybased programs, particularly in the area of vocational rehabilitation. Anecdotal reports of the inadequacy of vocational rehabilitation programs to address the needs of people with monocular vision indicate that this population's options for retaining jobs, retraining, and career development are being overlooked.

\section{Implications for practice}


On the basis of the results of this study, we can make several recommendations. First, information about the difficulties associated with monocular vision and their impact on daily activities should be provided at the time of diagnosis, when the possibility of losing one eye or significant vision in one eye is identified. Second, persons with monocular vision should be referred to appropriate community rehabilitation services on their discharge from the hospital to help them return to daily life. Third, these services should assist people with monocular vision and their families to develop the coping strategies needed to manage the effects of the impairment. In particular, rehabilitation interventions need to address the vocational needs of people with monocular vision through appropriate ergonomic workplace modifications and alternative work programs or career counseling. Fourth, the range of problems that may be associated with monocular vision suggests the need for an assessment tool to assist with the development of relevant rehabilitation programs. Finally, more support programs need to be established to address the ongoing issues experienced by people with monocular vision. Examples of such programs in Australia include the Guide Dogs Queensland Telelink/Support Group Program and the Monovision Group at the Royal Victorian Eye and Ear Hospital, both of which foster the sharing of experiences and the provision of emotional support among members.

\section{Limitations and recommendations for further}




\section{research}

A significant limitation of this study was that most of the participants were drawn from one rehabilitation agency, albeit the only provider of rehabilitation services to people with monocular vision in Queensland. These participants may not be representative of the wider population of people with monocular vision, many of whom may not experience problems associated with the condition. Unfortunately, this issue is compounded by the lack of data on the incidence and impact of monocular vision in Australia. Another limitation was that the study included only one person with congenital monocular vision, thereby making it difficult to draw any conclusions about the experiences of this population. Although statistics are not available in Australia on the incidence of congenital monocular vision, anecdotal evidence suggests that this population is much smaller than that of people with acquired monocular vision.

Further research is required to determine the incidence of monocular vision in Australia and to assess the level of understanding of the condition by providers of medical and rehabilitation services. Research is also needed on the range of individual factors that may affect the experience of monocular vision, such as age of onset, loss of the dominant versus the nondominant eye, gender, and jobs held before the injury. It is hoped that this research will help bring the needs of people with monocular vision to the attention of governments 
and service providers alike.

\section{References}

Brady, F. B. (1994). A singular view, the art of seeing with one eye (5th ed.). Ontario, Canada: Edgemore Enterprises.

Cooper, R. (2000). Magic eye: How to see 3D. Optometrists Network [Online]. Available: http:// www.vision3d.com

Gilberg, S. M. (1998). The artificial eye patient. University of Ottawa Eye Institute [Online]. Available: http://www.ohri.ca/ program/vision

Knoth, S. (1995). Monocular blindness: Is it a handicap? View, 26 , 177-180.

Linberg, J. V., Tillman, W. T., \& Allara, R. D. (1988). Recovery after loss of one eye. Ophthalmic Plastic Reconstructive Surgery, 4 , 135-138.

McKnight, A. J., Shinar, D., \& Hilburn, B. (1991). The visual and driving performance of monocular and binocular heavy-duty truck drivers . Accident Analysis and Prevention, 23 , 225-237.

Neuman, W. (1997). Social research methods:

Qualitative and quantitative approaches (3rd ed.). Boston: Allyn \& Bacon. 
O'Donnell, L. M., \& Smith, A. J. (1994). Visual cues for enhancing depth perception . Journal of Visual Impairment \& Blindness, 88, 258-266.

Patton, M. Q. (1990). Qualitative evaluation and research methods (2nd ed.). Newbury Park, CA: Sage.

Putman, S. H., \& Adams, K. M. (1992). Regressionbased prediction of long-term outcome following multidisciplinary rehabilitation for traumatic brain injury. Clinical Neuropsychologist, 6 , 383-405.

Reinharz, S., (1979). On becoming a social scientist . San Francisco: Jossey-Bass.

Schein, J. D. (1988). Acquired monocular disability. Journal of Visual Impairment \& Blindness, 82, 279281.

Schiff, W. (1980). Perception: An applied approach . Boston: Houghton Mifflin.

Servos, P., Goodale, M. A., \& Jakobson, L. S. (1992). The role of monocular vision in prehension: A kinematic analysis. Vision Research, 32, 1513-1521.

Spring, T. A. (1992). One-eyed view of life: An insight into the problems of monocular vision. Sydney, Australia: Author.

Nicholas Buys, Ph.D., professor, School of Human 
Services, Griffith University, Logan Campus, University Drive, Meadowbrook, Queensland 4131, Australia; e-mail: <n.buys@griffith.edu.au>.Jorge Lopez, MHS, client services, Guide Dogs Queensland, P.O. Box 50, Bald Hills, QLD 4036, Australia.

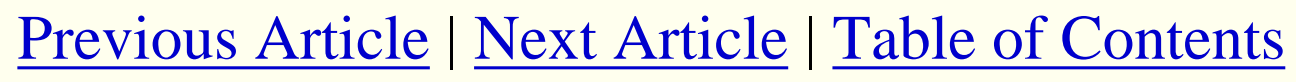

JVIB, Copyright (C) 2006 American Foundation for the Blind. All rights reserved.

\section{Search JVIB | JVIB Policies | Contact JVIB | Subscriptions | JVIB Home}

If you would like to give us feedback, please contact us at jvib@afb.net.

www.afb.org | Change Colors and More | Contact Us | Site Map | Site Search Go

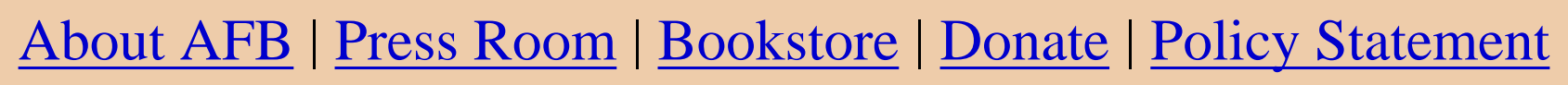

Please direct your comments and suggestions to afbinfo@afb.net Copyright (c) 2007 American Foundation for the Blind. All rights reserved. 